\title{
A Covid-19 e o desafio para o cuidado das populações vulneráveis no Estado do Paraná
}

\author{
Covid-19 and the challenge for care of vulnerable \\ populations in the State of Paraná
}

Lucimar Pasin de Godoy'1, Rosane Souza Freitas², Elaine Cristina Vieira de Oliveira ${ }^{3}$

\begin{abstract}
1. ORCID: http://orcid.org/0000-0002-1350-2099. Assistente Social, Especialista em saúde coletiva. Secretaria de Estado da Saúde do Paraná, Curitiba, Paraná, Brasil. E-mail: lucimargodoy@ sesa.pr.gov.br.

2. ORCID: http://orcid.org/0000-0003-3491-5770. Assistente Social, Mestre em Políticas Públicas. Secretaria de Estado da Saúde do Paraná, Curitiba, Paraná, Brasil. E-mail: rosane.freitas@ sesa.pr.gov.br.

3. ORCID: http://orcid.org/0000-0002-8484-5259. Nutricionista, Mestre em Alimentação e Nutrição. Secretaria de Estado da Saúde do Paraná, Curitiba, Paraná, Brasil. E-mail: elainevieira@ sesa.pr.gov.br.
\end{abstract}

CONTATO: Lucimar Pasin de Godoy | Rua Piquiri, n 170, Bairro Rebouças - Curitiba, Paraná - CEP 80230-140 | (41) 3330-4499 | E-mail: lucimarpasin@sesa.pr.gov.br / comunidades.spp@ sesa.pr.gov.br

RESUMO A desigualdade agrava a invisibilidade de comunidades vulneráveis. O Sistema Único de Saúde aplica o princípio da equidade, o qual considera as peculiaridades de cada pessoa para possibilitar o acesso à saúde de forma integral. No cenário da pandemia da Covid-19, a Secretaria de Estado da Saúde do Paraná implementou recomendações, orientações e estratégias de cuidado para populações vulneráveis. Este trabalho apresenta as ações desenvolvidas para mitigar os efeitos negativos da pandemia nas comunidades vulneráveis.

DESCRITORES: Equidade em saúde. Infecções por Coronavírus. Pandemias. Populações Vulneráveis.ação de seus impactos e redução das iniquidades em saúde.

ABSTRACT Inequality furthers the invisibility of vulnerable populations. The Sistema Único de Saúde (SUS, Unified Health System, the Brazilian health care 
system) applies the principle of equity, thus considering the peculiarities of each person to enable health access in an integral way. In the pandemic scenario of Covid-19, the Paraná State Department of Health implemented recommendations, guidelines and care strategies for vulnerable populations. This work presents the developed actions which mitigate the negative effects of the pandemic on vulnerable communities.

DESCRIPTORS: Health Equity. Coronavirus Infections. Pandemics. Vulnerable Populations.

\section{INTRODUÇÃO}

$\mathbf{E}$ m 30 de janeiro de 2020 a Organização Mundial da Saúde (OMS) declarou Emergência em Saúde Pública de Importância Internacional devido ao surto decorrente do novo coronavírus (SARS-CoV-2), e posteriormente caracterizou a Covid-19 como pandemia. Em poucos meses a doença atingiu diversos países, incluindo o Brasil. Devido à não existência de medicamentos eficazes para o tratamento da Covid-19, foram recomendadas medidas não farmacológicas para conter a transmissão do vírus na população. Em meio às orientações preconizadas nas normativas sanitárias, destacam-se o distanciamento e isolamento social, o uso de máscara e a frequente higienização das mãos com água e sabão ou álcool1-2.

No entanto, eseas medidas, aparentemente simples e acessíveis a toda a população, são praticamente inviáveis para alguns grupos. Isso expõe a desigualdade social, que é crônica no nosso país, sobretudo no que se refere às populações historicamente invisibilizadas e com acesso negado às políticas de saúde, habitação, saneamento e outras políticas de proteção social’3. No contexto destas populações vulneráveis estão os povos ciganos e indígenas, população quilombola, a do campo, da floresta e das águas, dentre outras comunidades tradicionais. Inclui-se também a população negra, as pessoas privadas de liberdade, em situação de rua, os migrantes, as lésbicas, gays, bissexuais, travestis e transexuais (LGBT - terminologia utilizada na política nacional4). 
O Paraná é subdividido em quatro Macrorregionais de Saúde (Figura 1), e estas populações vulneráveis vivem em todo o Estado, no entanto, alguns povos estão mais presentes em determinadas regiões. Verifica-se que a concentração de povos indígenas é maior nos municípios que compõem as macrorregiões Leste, Oeste e Norte; as comunidades quilombolas nos municípios das macrorregiões Leste e Oeste; e a população em situação de rua nos grandes centros urbanos.

Figura 1. Subdivisão do Paraná em Macrorregionais de Saúde.

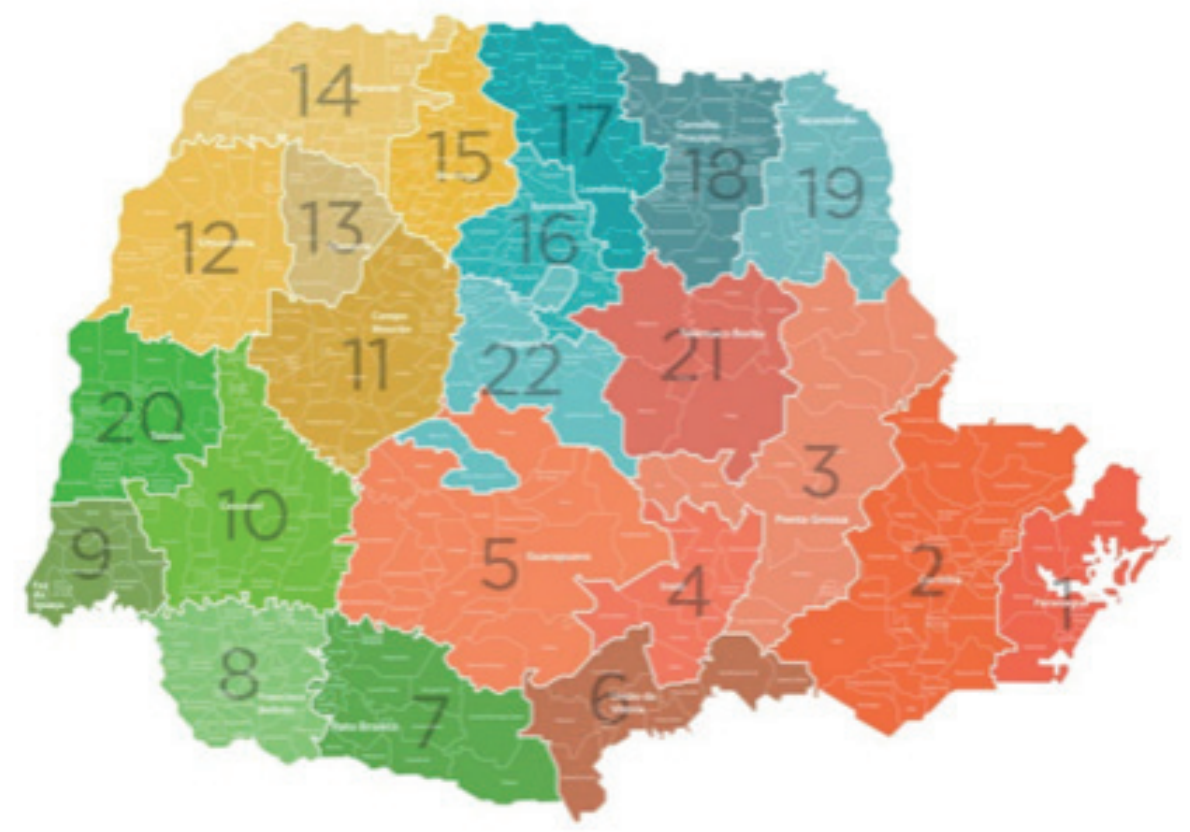

\section{SECRETARIA DA SAÚDE DO PARANÁ}

DIVISÃO POR REGIONAIS E MACRORREGIONAIS

\begin{tabular}{|c|c|c|c|c|c|}
\hline $\begin{array}{l}\text { REGIONAIS } \\
\text { DE saube }\end{array}$ & $\begin{array}{r}\text { Ne DE } \\
\text { Munacipios }\end{array}$ & $\begin{array}{l}\text { arcionais } \\
\text { De savide }\end{array}$ & $\begin{array}{r}\text { Ne be } \\
\text { munecipnos }\end{array}$ & $\begin{array}{l}\text { MACROREGIONLAIS } \\
\text { DE SAUDE }\end{array}$ & $\begin{array}{r}\text { NP DE } \\
\text { MUNicipios }\end{array}$ \\
\hline - PPARANAGUÁ & 7 & - BPCANCRTE. & n & - MaCro Leste & 93 \\
\hline - $2^{*}$ Curmena. & 29 & - Ier hapanuivar & -28 & - MACMO NOATE & 97 \\
\hline - 3 PONITA GPOSSA & Q & - is meneanch & 30 & a racho otstr & 24 \\
\hline - at Bati & 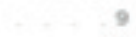 & - ve apucarana & $\pi$ & In Macho monotstre & ns \\
\hline - 5 GUARAPUMVA & $=20$ & - irlonogina & 21 & & \\
\hline - er unilo da vitoria & -9 & - ie confluo frocopio & 21 & & \\
\hline - "PATO BRANCO & -18 & - I9 jackezenoro & 22 & & \\
\hline - E RRANicisco BELTRLC & 27 & 202 TOLEDO & $\mathrm{z}$ & & \\
\hline - 9 FOZ dO IGUACO & 9 & - zr telenaco Bchea & & & \\
\hline - ne cascanel. & -25 & - 22 NAMPORA & 16 & & \\
\hline - III CAMPO MOURAO & $=25$ & - total do pacandí. & 390 & & \\
\hline - Q* UMUARAMA & $2 \pi$ & & & & \\
\hline
\end{tabular}

Fonte: SESA (2020). 
Considerando a necessidade de respostas oportunas para a contenção da evolução da doença nas comunidades vulneráveis que compõem a população atendida pelo Sistema Único de Saúde (SUS) Paraná, esta comunicação breve tem como objetivo explanar acerca das estratégias utilizadas pela Secretaria de Estado da Saúde do Paraná (SESA) para assegurar o princípio de equidade para as populações vulneráveis no atual momento de pandemia da Covid-19.

\section{RESULTADOS E DISCUSSÃO}

No contexto da pandemia e vulnerabilidade destaca-se o importante papel do SUS como uma política pública consolidada no Brasil. Esse sistema é a principal forma de acesso ao cuidado em saúde para a maior parcela dos grupos populacionais em vulnerabilidades. Dentre os princípios que o regem, vale destacar: a universalidade, que engloba o SUS como um direito de todos, sem distinção; a integralidade, que salienta a necessidade de assistência em saúde integral em todos os níveis de complexidade; e a equidade em saúde. Neste escopo, a equidade é entendida como:

[...] ofertar o cuidado, reconhecendo as diferenças nas condições de vida e saúde e de acordo com as necessidades das pessoas, considerando que o direito à saúde passa pelas diferenciações sociais e deve atender à diversidade. Ficando proibida qualquer exclusão baseada em idade, gênero, cor, crença, nacionalidade, etnia, orientação sexual, identidade de gênero, estado de saúde, condição socioeconômica, escolaridade ou limitação física, intelectual, funcional, entre outras, com estratégias que permitam minimizar desigualdades, evitar exclusão social de grupos que possam vir a sofrer estigmatização ou discriminação; de maneira que impacte na autonomia e na situação de saúde ${ }^{6: 6}$.

O princípio da equidade legitima o acesso do cuidado em saúde às populações vulneráveis, por considerar o contexto histórico, social e cultural, bem como as singularidades que vivem e que geram maior risco de adoecimento, em especial neste momento de pandemia. 
Diversos são os motivos que expõem as populações vulneráveis à contaminação pela Covid-19, como por exemplo a precária condição sanitária em que muitas pessoas vivem por falta de saneamento básico. Outro aspecto é a dificuldade em manter o distanciamento social pelas pessoas em privação de liberdade devido à superlotação nas unidades penais, ou pelos povos indígenas devido ao costume do viver coletivo. Destaca-se ainda a falta de moradia e a insegurança alimentar que acomete a população em situação de ruał:Diante de tal panorama, é fundamental considerar o princípio da equidade para que as populações vulneráveis possam ter seu direito à saúde efetivado. Nesse contexto, o Estado do Paraná, por meio do decreto n 4959, de 02 de julho de 2020, incluiu os povos indígenas e demais moradores de comunidades tradicionais no escopo das pessoas consideradas como integrantes do grupo de risco para a doença. Essa estratégia corrobora a importância desses grupos populacionais serem cuidados de acordo com suas especificidades.A articulação da SESA com todos os 399 municípios, por meio das 22 Regionais de Saúde (RS), tem o intuito de reafirmar o compromisso na efetivação do direito e atenção integral à saúde da população nesse momento de pandemia, incluindo as comunidades vulneráveis. A Secretaria de Estado, em consonância com o Ministério da Saúde e a OMS, construiu inúmeros documentos para respaldar os municípios na condução de estratégias de combate à Covid-19, sendo que estas orientações estão publicizadas no website da SESA.

No Plano de Contingência do Paraná - Covid-19 - Nível 3, foram incluídas recomendações direcionadas às comunidades vulneráveis. O plano é um documento elaborado com o intuito de nortear o Estado do Paraná no enfrentamento da pandemia e auxiliar os municípios na execução dos correspondentes Planos Municipais.

Devido ao cenário de transmissão da Covid-19, com necessidade de mobilização intensificada dos diversos atores, a SESA tem orientado os municípios que considerem as particularidades das populações vulneráveis no estabelecimento de fluxos assistenciais. O intuito é "[...] fortalecer as articulações intra 
e intersetoriais para garantir e ampliar o olhar e o acesso das comunidades vulneráveis $[\ldots]^{\prime \prime: 54 .}$

Dentre as orientações realizadas, destacamos algumas delas:

1- Incluir nos Planos de Contingência locais o cuidado em saúde das populações vulneráveis;

2- Garantir a oferta da vacina para influenza às populações vulneráveis;

3- Expandir o olhar sobre os agravos prevalentes na população negra (doença falciforme, hipertensão);

4- Incentivar que os Agentes Comunitários de Saúde realizem busca ativa das comunidades tradicionais em seus municípios, e que ofertem orientações de medidas de prevenção da disseminação da doença;

5- Articular com as políticas de Segurança Pública, Segurança Alimentar, Educação, Assistência Social, para o cuidado das populações vulneráveis no contexto atual;

6- Dialogar com a Política de Assistência Social a respeito da disponibilização de espaços apropriados para isolamento de pessoas em situação de rua com sintomas da doença;

7- Intensificar a parceria com o Distrito Sanitário Especial Indígena Litoral Sul (DSEI LSUL) e Departamento Penitenciário, para assegurar o cuidado dos povos indígenas e pessoas privadas de liberdade, respectivamente.

A SESA também desenvolveu um sistema de informação para a notificação de casos suspeitos da doença, sendo este denominado "Notifica Covid-19". Nesse sistema foram inseridos campos com dados sobre o paciente como, por exemplo, campo raça/cor e etnia, pessoa privada de liberdade, em situação de rua, dentre outros. Os dados oriundos desta ficha permitem traçar perfis epidemiológicos acerca da pandemia no Paraná, bem como tem possibilitado auxiliar na implementação de ações para o cuidado da população.

Dentre as experiências da SESA na condução das situações de transmissão do coronavírus em comunidades vulneráveis, destaca-se o enfrentamento do surto da Covid-19 na Aldeia Indígena de Ocoy, no município de São Miguel do Iguaçu, pertencente à $9^{\mathrm{a}} \mathrm{RS}$ - Foz do Iguaçu. O manejo da situação ocorreu por intermédio da direção e equipe técnica da referida regional, que protagoniza- 
ram as ações juntamente com representantes do município e do DSEI LSUL, Fundação Nacional Índio, Secretaria de Estado da Educação, Poder Judiciário, Ministério Público, Itaipu Binacional, Defesa Civil do Estado, Universidade Federal da Integração Latino-Americana e Universidade Estadual do Oeste do Paraná, além de lideranças da comunidade indígena local. O envolvimento de todos os parceiros foi fundamental para a adoção de medidas para a contenção do surto na aldeia.

\section{CONCLUSÃO}

As condições de vulnerabilidade às quais estão expostas algumas populações trouxeram à tona que certas medidas de enfrentamento à Covid-19 não são factíveis por alguns grupos. Observou-se que as estratégicas para nortear o cuidado das populações vulneráveis no Estado do Paraná foram ancoradas em ações de articulação e parcerias entre diversos atores, e que demandam a humanização do olhar para a oferta do cuidado, bem como o respeito às especificidades dos grupos.

Por meio dos diálogos e do compartilhamento de informações entre gestão da saúde do Estado do Paraná com os diversos profissionais de saúde que estão na linha de frente do enfrentamento da pandemia, é possível perceber o comprometimento das equipes e o envolvimento das mesmas no cuidado de toda a população.

Apesar do momento crítico do mundo, do Brasil e do Paraná, a mobilização em prol das populações vulneráveis tem sido uma janela de oportunidades para incluir, de maneira efetiva, estas comunidades como parte do território, possibilitando a consolidação de ações de promoção da saúde e prevenção de agravos, e dos princípios do SUS.

Assim, aqui apresentamos ações implementadas pelo Estado do Paraná para mitigar efeitos a curto, médio e longo prazos da pandemia nas comunidades 


\title{
mais vulneráveis.
}

\section{AGRADECIMENTOS}

\author{
Agradecemos à Profa. Dra. Elaine Drehmer de Almeida Cruz e à Laila Ra- \\ quel Pasin e Matos pelas contribuições ao texto.
}

\section{REFERÊNCIAS}

1. Brasil. Ministério da Saúde, Gabinete do Ministro. Portaria $n^{\circ} 188$, de 3 de fevereiro de 2020. Declara Emergência em Saúde Pública de importância Nacional (ESPIN) em decorrência da Infecção Humana pelo novo Coronavírus (2019-nCoV) [Internet]. Brasil: Diário Oficial da União; 2020 Fev. [citado em 2020 Jul 13]. Disponível em: https://www.in.gov.br/en/web/ dou/-/portaria-n-188-de-3-de-fevereiro-de-2020-241408388.

2. Governo do Estado do Paraná. Decreto n 4230, de 16 de março de 2020. Dispõe sobre as medidas para enfrentamento da emergência de saúde pública de importância internacional decorrente do Coronavírus - Covid-19. [Internet]. Paraná: 2020 Mar. [citado em 2020 Jul 13]. Disponível em: http://www.aen.pr.gov.br/arquivos/Decreto_4230.pdf.

3. Campello T, Gentili P, Rodrigues M, Hoewell GR. Faces da desigualdade no Brasil: um olhar sobre os que ficam para trás [Internet]. Rio de Janeiro: Saúde Debate; 2018 Nov. [citado em 2020 Jul 13]. Disponível em: http://dx.doi.org/10.1590/0103$11042018 s 305$.

4. Ministério da Saúde, Gabinete do Ministro. Portaria $n^{\circ}$ 2.836, de 1 de dezembro de 2011. Institui, no âmbito do Sistema Único de Saúde (SUS), a Política Nacional de Saúde Integral LGBT [Internet]. Brasil: 2011 Dez. [citado em 2020 Out 5]. Disponível em: http://bvsms.saude.gov.br/bvs/saudelegis/gm/2011/prt2836_01_12_2011.html.

5. Stopa SR, Malta DC, Monteiro CN, Szwarcwald CL, Goldbaum M, Cesar CLG. Acesso e uso de serviços de saúde pela população brasileira, Pesquisa Nacional de Saúde 2013 [Internet]. São Paulo: Rev Saúde Pública; 2017 Jun. [citado 2020 Jul 15]. Disponível em: http://dx.doi.org/10.1590/s1518-8787.2017051000074.

6. Brasil. Ministério da Saúde, Gabinete do Ministro. Portaria n 2.436, de 21 de setembro de 2017. Aprova a Política Nacional de Atenção Básica no âmbito do Sistema Único de Saúde (SUS) [Internet]. Brasil: 2017 Set. [citado em 2020 Jul 13]. Disponível em: https://bvsms.saude.gov.br/bvs/saudelegis/gm/2017/prt2436_22_09_2017.html.

7. Stevanim LF. Vulnerabilidades que aproximam. N 212. [lugar desconhecido]: Radis; 2020 Mai; 10-15.

8. Governo do Paraná. Decreto n 4959, de 16 de março de 2020. Acresce dispositivos ao Decreto n 4.230. [Internet]. Paraná: 2020 Mar. [citado em 2020 Jul 13]. Disponível em: https://www.legisweb.com.br/legislacao/?id=398022.

9. Secretaria de Estado da Saúde do Paraná. Plano de Contingência do Paraná COVID-19 (Nível 3 - Execução). [Internet]. Paraná: SESA; 2020 Set. [citado em 2020 Out 05]. Disponível em: https://www.saude.pr.gov.br/sites/default/arquivos restritos/files/documento/2020-10/Plano\%20FINAL\%2001.10.pdf.

RECEBIDO: $31 / 07 / 2020$

ACEITO: 07/10/2020 